\title{
Frontal fibrosing alopecia and extrafacial lichen planus pigmentosum in a caucasian woman*
}

\author{
Monserrat Franco-Muñoz ${ }^{1}$ \\ Belén Lozano-Masdemont ${ }^{1}$
}

\author{
Monica García-Arpa ${ }^{1}$ \\ Claudia Ramos-Rodríguez ${ }^{2}$
}

DOI: http:/ /dx.doi.org/10.1590/abd1806-4841.20176638

\begin{abstract}
We present the case of a 72-year-old Caucasian woman with frontal fibrosing alopecia of one year's duration. Eighteen months later, she presented with sudden hyperpigmentation on the submental area, neck and upper chest. Histopathological examination was consistent with lichen planus pigmentosus. This is the report of a unique case of a FFA and extrafacial lichen planus pigmentosus.
\end{abstract}

Keywords: Alopecia; Hyperpigmentation; Lichen planus

\section{INTRODUCTION}

Frontal fibrosing alopecia (FFA) is a lymphocytic cicatricial alopecia that shares common histophalologic features with lichen planopilaris. FFA can be associated with cutaneous or mucosal lichen planus, and recently with lichen planus pigmentosum (LPP). This association was first reported by Dlova in 2013 in south African patients. ${ }^{1}$ Most cases are women, with dark skin and LPP with facial involvement.

\section{CASE REPORT}

A 72-year-old, Caucasian, phototype II female presented with scalp hair loss, with progressive frontotemporal hairline recession and diffuse alopecia of eyebrows and eyelashes of one year's duration (Figure 1). She had been using antihypertensive medication (enalapril) for the previous 10 years. Routine laboratory investigations were normal including thyroid function and auto- immune markers. FFA was diagnosed and topical corticosteroids and hydroxychloroquine $200 \mathrm{mg}$ /day were prescribed but were discontinued four months later due to gastric intolerance. After 18 months, she developed sudden hyperpigmentation and mild itch on the neck region. On physical examination, gray-brown pigmented macules with a smooth surface that had become confluent, forming pigmented patches with a reticular pattern on the submental area, anterior and lateral regions of the neck and upper chest, were seen (Figure 2). There was no oral or nail involvement. She denied taking any medications, sun exposure or application of topical substances. Dermoscopic features were a pseudo-network pattern and areas with gray dots arranged in circles, without telangiectasias or other vascular images (Figure 3). Histopathological examination revealed epidermal atrophy, foci of basal vacuolar change and interface lichenoid reaction, isolated necrotic keratinocytes and abun-

\footnotetext{
Study submitted in 24.10 .2016

Approved by the Advisory Board and accepted for publication on 13.01.2017

* Work performed at the Hospital General Universitario Ciudad Real - Ciudad Real, Spain.

Financial Support: None.

Conflict of Interests: None.

1 Department of Dermatology, Hospital General Universitario Ciudad Real - Ciudad Real, Spain.

2 Department of Pathology, Hospital General Universitario Ciudad Real - Ciudad Real, Spain.
} 
dant melanophages in upper dermis, consistent with LPP (Figure 4). Photoprotection and topical corticosteroids were prescribed with poor response.

\section{DISCUSSION}

LPP is an uncommon variant of lichen planus, that tends to occur in middle-aged individuals with darkly pigmented skin. Clinically, lesions appear as small, ill-defined oval to round macules, which may be discrete or become confluent to form large patches. The pigmentation varies from slate gray to dark brown. It primarily affects sun-exposed areas. Different clinical patterns of pigmentation including blotchy, reticular, perifollicular and diffuse (most common) and atypical variants like inverse, linear, mucosal and zosteriform have been described. ${ }^{2}$ Histopathologic features include epidermal atrophy, interface lichenoid dermatitis, vacuolar degeneration of the basal layer and pigment incontinence. On the other hand, the coexistence of FFA and LPP is rare and was recently

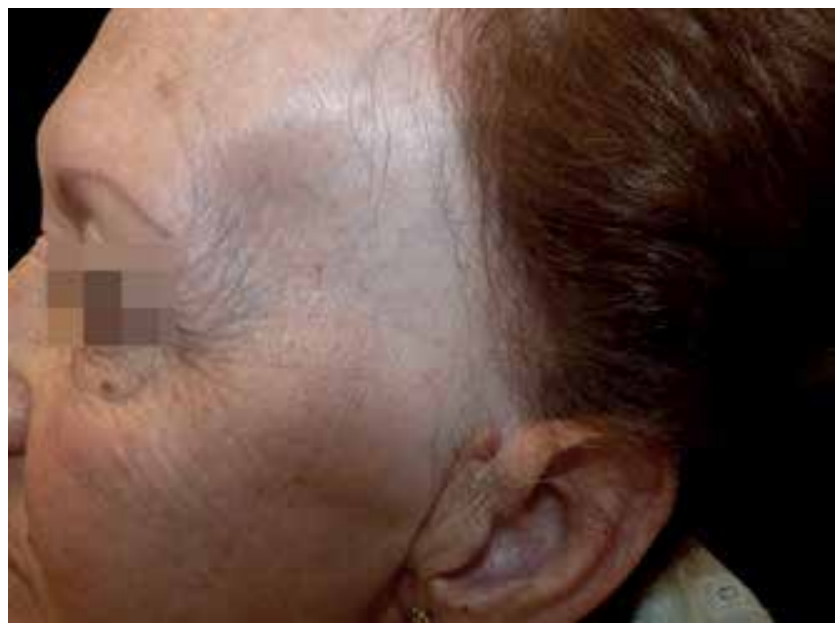

Figure 1: Alopecia over the frontotemporal region and loss of eyebrow

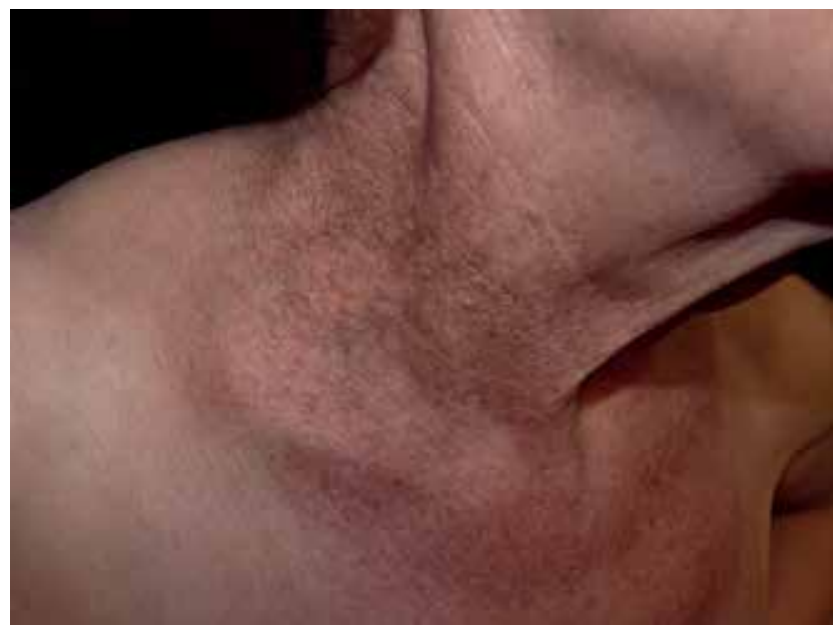

FIGURE 2: Reticular hyperpigmentation on the neck and upper chest region described. Up to date, 64 patients have been reported with two series of 24 and 37 patients and isolated cases; 62 patients were women, mostly postmenopausal with dark skin and there were only three cases with skin phototype III. ${ }^{1-4}$ All patients presented facial LPP and nearly one third had other sun-exposed area involvement, mainly the neck.

LPP usually precedes FFA, although sometimes the onset is not accurate and more rarely they are simultaneous. ${ }^{3}$ This initially led to the thought that LPP could be a premonitory sign of FFA, but this is unclear and both can also have very subtle changes in the initial stages and be overlooked. Four dermatoscopic patterns of LPP in patients with FFA have been described recently but without correlation with the clinical type of pigmentation, and they can coexist in the same patient: pseudo-network, speckled blue-grey dots (both patterns possibly correlate with prominent interfollicular interface dermatitis), dotted pattern and blue-grey dots arranged in circles (from injury to eccrine glands and follicular structures, respective-

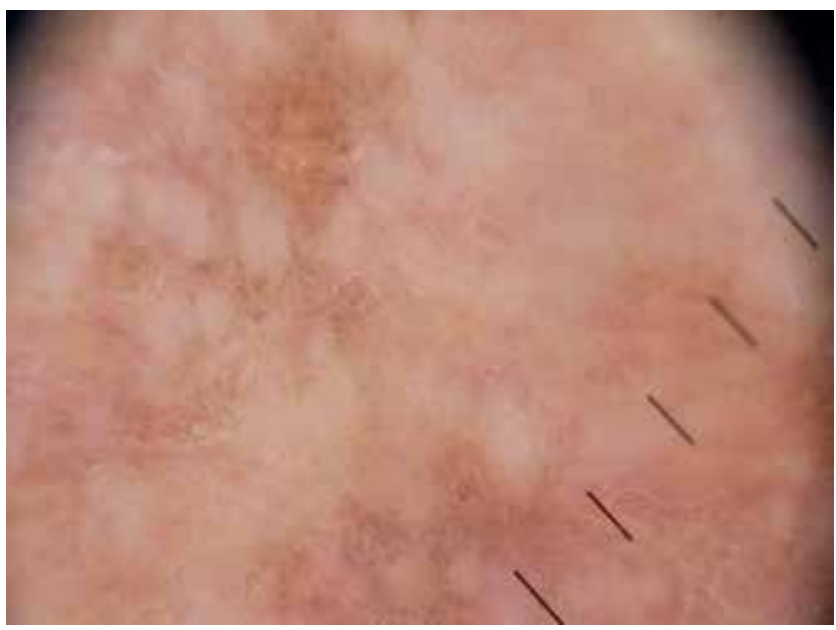

Figure 3: Dermoscopic image of lichen planus pigmentosus with pseudo-network pattern
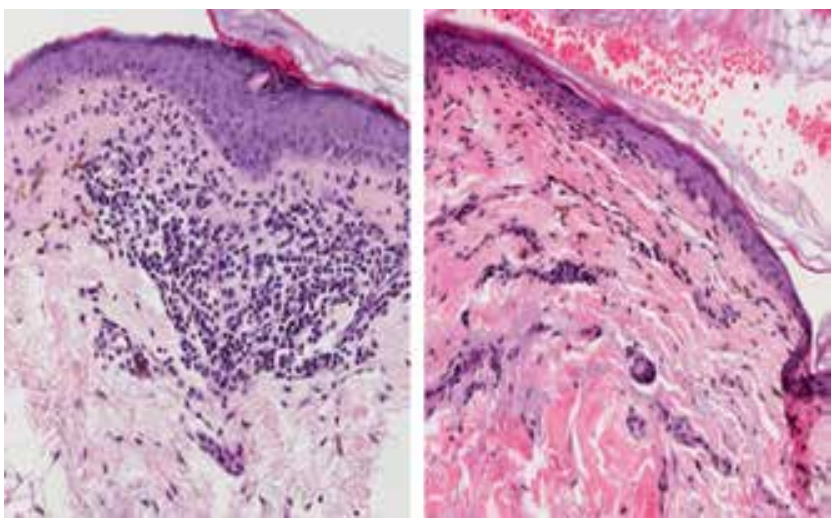

FigURE 4: A - Slightly atrophic epidermis with effacement of rete ridges. In the dermis, there is a lichenoid reaction and melanophages (Hematoxylin \& eosin, X20). B - Foci of vacuolar changes of the dermo-epidermal interface and abundant melanophages deeper in dermis than in postinflammatory pigmentation (Hematoxylin \& eosin, x20) 
ly). Vascular changes might be found in the context of an inflammatory process. ${ }^{3}$

The differential diagnosis of LPP should be established with a group of hyperpigmentation disorders, such as melasma, exogenous ochronosis, Riehl's melanosis and erythema dyschromicum perstans (EDP) or ashy dermatosis. LPP and EDP share many clinical and histopathologic similarities. ${ }^{5}$ EDP usually affects the trunk and upper extremities and presents melanin deposits mainly in deeper dermis, unlike LPP, which is located in the superficial dermis. Pigmentation from antimalarial is another differential diagnosis because it is a common treatment of FFA, occurs in $10-25 \%$ of patients, more frequently by chloroquine than hydroxychloroquine (35\% vs $13 \%$ ). The lesions are typically blue-grey macules that enlarge and become confluent with variable distribution affecting: head, trunk, extremities and areas not exposed to the sun, including mucosal areas. Pigmentation onset ranges from four months to 22 years following the initiation of therapy, usually at high doses and the treatment consisted of discontinuing the antimalarial drug, which led to a gradual decrease in the hyperpigmentation within several months. ${ }^{7}$ The histopathology consists of yellow to dark brown granules with- in macrophages and extracellularly in the dermis without inflammatory phase and damage to the dermo-epidermal junction.

Neither of the two entities have curative treatment. Photoprotection is essential in the LPP and other therapies include topical corticosteroids and tacrolimus, laser and oral retinoids, usually with poor results, although better response to treatment is observed in patients with a shorter duration ( $\leq 5$ years) of disease and limited body area involvement. ${ }^{8}$

In conclusion, we report another case of FFA with LPP. This is interesting because the patient is a Caucasian woman with a clear history of FFA preceding LPP, unlike most previously reported cases. Furthermore, this is the first case in which the LPP does not affect the face. In the presence of one of these entities, we believe it is important to consider this association, which is probably underdiagnosed. Early diagnosis of FFA is important to allow treatment with the intention of slowing or preventing progression, and although there are several therapeutic options for LPP, the results are usually unsatisfactory, and it seems to be better response in patients with shorter duration.]

\section{REFERENCES}

1. Dlova NC. Frontal fibrosing alopecia and lichen planus pigmentosus: is there a link? Br J Dermatol. 2013;168:439-42.

2. Rao R, Sarda A, Khanna R, Balachandran C. Coexistence of frontal fibrosing alopecia with lichen planus pigmentosus. Int J Dermatol. 2014;53:622-4.

3. Pirmez R, Duque-Estrada B, Donati A, Campos-do-Carmo G, Valente NS, Romiti $\mathrm{R}$, et al. Clinical and dermoscopic features of lichen planus pigmentosus in 37 patients with frontal fibrosing alopecia. Br J Dermatol. 2016;175:1387-90.

4. Berliner JG, McCalmont TH, Price VH, Berger TG. Frontal fibrosing alopecia and lichen planus pigmentosus. J Am Acad Dermatol. 2014;71:e26-7.

5. Rieder E, Kaplan J, Kamino H, Sanchez M, Pomeranz MK. Lichen planus pigmentosus. Dermatol Online J. 2013;19:20713.

6. Mir A, Boyd KP, Meehan SA, McLellan B. Hydroxycholoroquine-induced hyperpigmentation. Dermatol Online J. 2013;19:20723.

7. Puri PK, Lountzis NI, Tyler W, Ferringer T. Hydroxychloroquine-induced hyperpigmentation: the staining pattern. J Cutan Pathol. 2008;35:1134-7.

8. Muthu SK, Narang T, Saikia UN, Kanwar AJ, Parsad D, Dogra S. Low-dose oral isotretinoin therapy in lichen planus pigmentosus: an open-label non-randomized prospective pilot study. Int J Dermatol. 2016;55:1048-54.
MAILING ADDRESS:

Monserrat Franco-Muñoz

Servicio de Dermatología,

Hospital General Universitario Ciudad Real.

C/Obispo Rafael Torija, s/n,

13005 Ciudad Real, Spain.

E-mail: monse.franco.m@gmail.com

How to cite this article: Franco-Muñoz M, García-Arpa M, Lozano-Masdemont B, Ramos-Rodríguez C. Frontal fibrosing alopecia and extrafacial lichen planus pigmentosum in a Caucasian woman. An Bras Dermatol. 2017;92(5 Suppl 1): 76-8. 